\title{
Impacto da obesidade na saúde bucal: revisão de literatura
}

\author{
Impact of obesity on oral health: a literature review
}

\author{
Letícia Ferreira de Freitas Brianezzi* \\ Luana Polioni Al-Ahj* \\ Letícia A. Prestes* \\ Lígia M. Andreatta* \\ Layla R. M. Vasconcelos* \\ Juliane Avansini Marsicano* \\ Arsenio Sales-Peres ${ }^{* *}$ \\ Sílvia Helena de Carvalho Sales Peres ${ }^{* * *}$
}

\section{Resumo}

As taxas de prevalência e de incidência referentes à obesidade têm se tornado alarmantes, tanto em adultos como em crianças. O acúmulo excessivo de gordura no organismo pode acarretar prejuízos à saúde dos indivíduos. Objetivo: o presente estudo tem como objetivo apresentar uma revisão de literatura sobre a interação da obesidade com a saúde bucal por meio de um estudo ordenado em periódicos de circulação nacional e internacional. Materiais e método: foram encontrados 514 estudos, dentre os quais, foram selecionados 18 artigos, com base nos seguintes critérios: época de publicação, tema adequado ao proposto neste trabalho, ideias claras, objetivas e condizentes com o título do estudo. Resultados: os estudos selecionados mostraram associações entre doença periodontal e obesidade. Já em relação aos trabalhos que associavam cárie dental e obesidade, houve algumas divergências, estando estas em minoria. Conclusão: conclui-se que os pacientes obesos necessitam de atenção em saúde bucal, especialmente em relação à prevenção da doença periodontal e cárie dentária.

Palavras-chave: Saúde bucal. Obesidade. Periodontite. Cárie dentária.

\section{Introdução}

A obesidade é uma doença crônica caracterizada pelo acúmulo excessivo de gordura corporal, proporcionalmente à massa magra ${ }^{1,2}$. $\mathrm{O}$ sobrepeso, segundo a Organização Mundial da Saúde ${ }^{3}$, é considerado um índice de massa corporal variando de 25 a 29,9 $\mathrm{kg} / \mathrm{m}^{2}$, e a obesidade, como um índice de massa corporal $>30 \mathrm{~kg} / \mathrm{m}^{24}$.

A prevalência da obesidade está aumentando tanto em países desenvolvidos como nos subdesenvolvidos. Esse aumento pode ter resultado da transformação no estilo de vida da sociedade moderna, a qual alterou, principalmente, os hábitos alimentares com o aumento do consumo de alimentos processados e o desencorajamento de atividades físicas, levando a um excesso calórico e ao sedentarismo, favorecendo, assim, a obesidade ${ }^{4}$. A prevalência em crianças também está aumentando, e isso pode ser devido a fatores comportamentais, envolvendo educação familiar, visto que crianças com pais obesos têm cerca de $80 \%$ mais chance de serem obesas ${ }^{5}$. A obesidade e o sobrepeso favorecem o aparecimento de comorbidades, como doenças cardíacas, hipertensão arterial, osteoartrite, diabetes tipo 2 e al-

\footnotetext{
Graduanda da Faculdade de Odontologia de Bauru da Universidade de São Paulo (USP). Bauru, SP, Brasil.

* Mestre em Odontologia em Saúde Coletiva, Departamento de Odontopediatria, Ortodontia e Saúde Coletiva da Faculdade de Odontologia de Bauru da Universidade de São Paulo (USP). Bauru, SP, Brasil.

*** Professor associado do Departamento de Odontopediatria, Ortodontia e Saúde Coletiva da Faculdade de Odontologia de Bauru da Universidade de São Paulo (USP). Bauru, SP, Brasil.
} 
guns tipos de câncer ${ }^{6}$, além de relacionarem-se com doença periodontal e cárie, sendo estas abordadas neste estudo.

A relação entre a obesidade e a saúde bucal pode corresponder às doenças orais infecciosas, como cárie dentária e doença periodontal, e ao impacto causado na capacidade mastigatória, levando a mudanças, como a troca de alimentos ricos em nutrientes por ricos em açúcares e gorduras saturadas. Com efeito, a obesidade é, muitas vezes, marcada por uma desequilibrada dieta rica em açúcares, que estimulam o crescimento de bactérias cariogênicas e favorecem o desenvolvimento da lesão de cárie ${ }^{7-9}$.

Estudo sugere que a falta de dentes e lesões cariosas estão relacionadas com aumento do índice de massa corporal (IMC) ${ }^{7}$. Uma revisão dos guias dietéticos internacionais lançados desde a década de 1960 demonstrou que a maioria $(84,5 \%)$ recomenda a redução do consumo de açúcares, tendo como objetivo a prevenção de várias doenças crônicas, principalmente a cárie e a obesidade. A redução do consumo de açúcar constitui, portanto, uma importante medida de promoção de saúde bucal e geral, tendo como estratégia vital a educação em saúde ${ }^{9}$.

A doença periodontal é uma doença infectoinflamatória que acomete os tecidos de suporte (gengiva) e sustentação (cemento, ligamento periodontal e osso) dos dentes. A etiologia é microbiana, sendo agravada quando a higiene bucal é negligencia$\mathrm{da}^{6,10}$. Há estudos ${ }^{6,10}$ demonstrando que talvez seja a resistência à insulina que regula a relação entre a obesidade e a doença periodontal. Descobriu-se, também, que os indivíduos com IMC elevado produzem um nível mais alto de proteínas inflamatórias $^{6,10}$. Resultados de um estudo de coorte realizado em Pelotas sugerem que gengivite em dois ou mais dentes está relacionada à obesidade, e essa associação pode relacionar-se, também, com a higiene oral e inflamação sistêmica de baixo grau. No que se refere ao cálculo dentário, a circunferência da cintura e a obesidade apresentaram taxas de prevalência de magnitude similar. Entretanto, a presença de bolsas periodontais não esteve relacionada com a obesidade nem com a circunferência da cintura ${ }^{11}$.

É importante ressaltar que a obesidade, por si só, não pode ser usada como o único indicador de saúde oral, sendo necessário considerar outros fatores, como a condição socioeconômica, a higiene bucal, hábitos alimentares, entre outros ${ }^{7}$.

A relação entre o estado nutricional e a saúde bucal ainda é controversa na literatura científica ${ }^{7}$. $\mathrm{O}$ presente trabalho tem como objetivo proceder a uma revisão literária dos estudos envolvendo obesidade relacionada à doença periodontal e à cárie dental.

\section{Materiais e método}

Foi realizada uma revisão dos artigos relacionando a saúde bucal com a obesidade. Entre as doenças que afetam a qualidade da saúde bucal relacionadas ao excesso de massa corpórea, este estudo deu prioridade para a doença periodontal e a cárie dentária, por serem mais prevalentes na população.

A base de dados Medline (PubMed, SciELO e Bireme) foi utilizada para identificar os estudos que avaliaram a interação da saúde bucal e obesidade. Os descritores utilizados para a busca foram: oral health AND obesity doença periodontal AND obesidade periodontal disease AND obesity cárie dentária AND obesidade e dental caries AND obesity.

Os critérios adotados para a inclusão e exclusão neste estudo foram: ter sido publicado entre 1997 e 2010, estar escrito em inglês ou português, abordar tema adequado ao aqui proposto, relatar estudo em seres humanos, conter ideias claras, objetivas e condizentes com o título do trabalho. Os artigos que não obedeceram a esses critérios não foram selecionados. Apenas estudos primários foram incluídos, correspondendo a ensaios clínicos randomizados, ensaios clínicos não randomizados, coorte ou caso controle. Cinco revisores selecionaram os estudos que cumpriram os critérios de inclusão. Inicialmente, os estudos foram selecionados por título e resumo; apenas quando não havia informação suficiente no título e no resumo para permitir uma decisão clara, foram obtidos os estudos na íntegra.

Após a seleção dos estudos, os cinco revisores avaliaram todos os estudos selecionados na íntegra para obter as informacões necessárias (amostra estudada, tipo de estudo, índices utilizados e os principais resultados).

\section{Resultados}

Depois da análise de todas as bases de dados, chegou-se a um número total de 514 trabalhos encontrados na literatura científica. No início da revisão, todos os artigos foram selecionados, porém, após uma leitura sucinta dos títulos e resumos em geral, apenas 17 pesquisas científicas foram consideradas pertinentes, por investigarem a relação da obesidade com a saúde bucal. Os trabalhos encontram-se descritos no Quadro 1. 


\begin{tabular}{|c|c|c|c|c|}
\hline Autores (ano) & $\begin{array}{l}\text { Amostra } \\
\mathrm{n} \text { (idade) }\end{array}$ & Tipo de estudo & Índices & Resultados \\
\hline Peréz LS et al. ${ }^{12}$ (2010) & $\begin{array}{l}110 \\
(7-11)\end{array}$ & Longitudinal & $\begin{array}{c}\mathrm{IMC} / \\
\mathrm{CPOD} / \mathrm{CPOS}\end{array}$ & $\begin{array}{l}\text { Crianças acima do peso apresentaram maior número } \\
\text { de dentes permanentes e menor experiência de cárie. }\end{array}$ \\
\hline Cinar e Murtomaa ${ }^{13}$ (2010) & $\begin{array}{c}611 \\
(10-12)\end{array}$ & Transversal & $\begin{array}{c}\mathrm{IMC} \\
\mathrm{CPOD} / \mathrm{IPC}\end{array}$ & $\begin{array}{l}\text { A obesidade esteve associada à cárie dentária, en- } \\
\text { quanto a desnutrição, à doença periodontal. Verifi- } \\
\text { cou-se, também, a relação com as doenças bucais e } \\
\text { a condição socioeconômica. }\end{array}$ \\
\hline Andrade et al. ${ }^{14}$ (2009) & $\begin{array}{c}887 \\
(60-96)\end{array}$ & Transversal & $\begin{array}{c}\mathrm{IMC/} \\
\mathrm{CPOD} / \text { dentes } \\
\text { posteriores em oclusão }\end{array}$ & $\begin{array}{l}\text { Não houve associação entre obesidade e cárie den- } \\
\text { tária, porém, verificou-se relação entre a redução de } \\
\text { ingestão de nutrientes e perda dentária. }\end{array}$ \\
\hline Goodson et al. ${ }^{15}$ (2009) & $\begin{array}{c}723 \\
(20-45)\end{array}$ & Transversal & IMC & $\begin{array}{l}\text { Os pacientes com sobrepeso apresentaram mais bac- } \\
\text { térias na saliva, podendo haver associação com a } \\
\text { doença periodontal. }\end{array}$ \\
\hline Haffajee et al. ${ }^{16}$ (2009) & $\begin{array}{c}695 \\
(48,8)\end{array}$ & & $\begin{array}{l}\text { IMC } \\
\text { ORs } \\
\text { BOP }\end{array}$ & $\begin{array}{l}\text { Pacientes obesos e com sobrepeso apresentaram } \\
\text { maior número da bactéria T. forsythia, o que aumenta } \\
\text { a probabilidade de apresentarem a doença periodon- } \\
\text { tal e haver uma progressão mais rápida da doença. }\end{array}$ \\
\hline Jürgensen et al. ${ }^{17}$ (2009) & $\begin{array}{l}621 \\
(12)\end{array}$ & Transversal & $\begin{array}{l}\text { IMC } \\
\text { CPOD }\end{array}$ & $\begin{array}{l}\text { Não houve associação entre IMC e doenças bucais } \\
\text { ou fatores de risco comuns. }\end{array}$ \\
\hline Khader et al. ${ }^{18}$ (2009) & $\begin{array}{c}340 \\
(18-70)\end{array}$ & Transversal & $\begin{array}{l}\text { IMC/CC/\% de teor de } \\
\text { gordura/BOP/AL }\end{array}$ & $\begin{array}{l}\text { A obesidade calculada a partir do IMC, CC, porcen- } \\
\text { tagem de teor de gordura teve associação com o au- } \\
\text { mento das chances de desenvolver periodontite. }\end{array}$ \\
\hline Kongsted et al. ${ }^{19}$ (2009) & $\begin{array}{c}1597 \\
(20-95)\end{array}$ & Transversal & $\begin{array}{l}\mathrm{AL}, \mathrm{SS}, \mathrm{BOP} \\
\mathrm{IMC}\end{array}$ & $\begin{array}{l}\text { O IMC não esteve relacionado com o aumento do } \\
\text { AL, porém, apresentou associação significante com } \\
\text { o aumento do BOP. }\end{array}$ \\
\hline Kushiama et al. ${ }^{20}$ (2009) & $\begin{array}{c}170 \\
(40-70)\end{array}$ & Transversal & $\begin{array}{l}\text { IPC } \\
\text { IMC }\end{array}$ & $\begin{array}{l}\text { Não encontraram relação entre síndrome metabólica } \\
\text { e a presença da doença periodontal. }\end{array}$ \\
\hline Morita et al. ${ }^{21}$ (2009) & $\begin{array}{l}2478 \\
(24-60)\end{array}$ & Transversal & OR/ & $\begin{array}{l}\text { Verificaram associação entre a presença da doença } \\
\text { periodontal e a síndrome metabólica. }\end{array}$ \\
\hline Tramini et al. ${ }^{22}$ (2009) & $\begin{array}{l}835 \\
(12)\end{array}$ & Transversal & IMC/CPOD & $\begin{array}{l}\text { Não encontraram associação entre IMC e CPOD ele- } \\
\text { vado. }\end{array}$ \\
\hline Ekuni et al. ${ }^{23}$ (2008) & $\begin{array}{c}618 \\
(18-24)\end{array}$ & Transversal & $\begin{array}{l}\text { IMC/ } \\
\text { IPC }\end{array}$ & $\begin{array}{l}\text { Constataram que a obesidade pode ser um fator de } \\
\text { risco para o desenvolvimento e progressão da perio- } \\
\text { dontite entre indivíduos jovens. }\end{array}$ \\
\hline Hilgert et al. ${ }^{24}(2008)$ & $\begin{array}{l}872 \\
(>65)\end{array}$ & Transversal & $\begin{array}{c}\text { IMC } \\
\text { Uso de prótese }\end{array}$ & $\begin{array}{l}\text { A obesidade esteve associada a uma condição bucal } \\
\text { ruim (edentulismo/ausência de reabilitação oral). }\end{array}$ \\
\hline Ngatia et al. ${ }^{25}$ (2008) & $\begin{array}{c}289 \\
(>45)\end{array}$ & Transversal & IMC/CPOD & $\begin{array}{l}\text { Concluíram que tanto a desnutrição como a obesi- } \\
\text { dade estão relacionadas com problemas dentários } \\
\text { (cárie e mobilidade dentária). }\end{array}$ \\
\hline Moreira et al. ${ }^{8}$ (2006) & $\begin{array}{c}3330 \\
(12-15)\end{array}$ & Transversal & IMC/CPOD & $\begin{array}{l}\text { Não existiram associações entre cárie dentária e obe- } \\
\text { sidade. }\end{array}$ \\
\hline Östberg et al. ${ }^{26}$ (2007) & $\begin{array}{c}2018 \\
(30-74)\end{array}$ & Transversal & IMC & $\begin{array}{l}\text { Verificou-se associação entre a perda dentária e a } \\
\text { obesidade. }\end{array}$ \\
\hline Yöslato et al. ${ }^{27}$ (2008) & $\begin{array}{c}2841 \\
(30-49\end{array}$ & Transversal & IMC & $\begin{array}{l}\text { Houve associação entre a obesidade e a presença da } \\
\text { doença periodontal. }\end{array}$ \\
\hline
\end{tabular}

Quadro 1 - Revisão da literatura acerca de interação entre obesidade e saúde bucal

IMC - índice de massa corporal

CPOD - cariado, perdido, obturado - dente

CPOS- cariado, perdido, obturado - superfície

IPC - índice periodontal comunitário

ORs/OR - perda óssea
BOP - bolsa óssea periodontal

$\mathrm{AL}$ - inserção periodontal

$\mathrm{SS}$ - índice de sangramento gengival

CC - circunferência da cintura 


\section{Discussão}

A obesidade pode provocar o aparecimento de outras doenças que agravam o estado de saúde do paciente, trazendo, assim, prejuízos à saúde sistêmica, com possibilidade de comprometer a saúde bucal ${ }^{5}$.

No estudo de Saito et al. ${ }^{28}$, com adultos japoneses, o aumento do índice de massa corporal e razão cintura/quadril foi associado com o risco crescente de periodontite. Da mesma forma, Al-Zharani et al. ${ }^{29}$ analisaram dados do Terceiro Inquérito $\mathrm{Na}$ cional de Saúde e Nutrição (Third National Health and Nutrition Examination Survey) e relataram uma associação significativa nas medidas de gordura corporal e doença periodontal entre os adultos mais jovens, porém não entre os de meia-idade ou idosos. Usando o mesmo banco de dados, Wood et al. ${ }^{30}$ avaliaram a relação entre diferentes medidas de adiposidade e doença periodontal e verificaram a necessidade de mais estudos do tipo longitudinal. Khader et al. ${ }^{18}$ (2009) também utilizaram o índice de massa corpórea, circunferência de cintura e porcentagem de gordura, relatando uma associação significativa à maior chance de ter periodontite.

Bezerra et al. ${ }^{6} \mathrm{e}$ Khader et al. ${ }^{18}$ demonstraram que a obesidade eleva o risco de doença periodontal e que isso pode estar associado com a resistência à insulina e os altos níveis de proteínas inflamatórias produzidas. Indivíduos com índice de massa corporal elevado produzem um nível mais alto de proteínas inflamatórias, portanto, o tecido adiposo serve como um reservatório de citocinas inflamatórias, sendo possível que o aumento de gordura corporal aumente a propensão de um anfitrião ativo e esteja presente na resposta inflamatória da doença periodontal. Isso porque a atividade imunológica de tecido adiposo pode desempenhar um papel importante tanto no desenvolvimento de resistência à insulina quanto na doença periodontal. Além disso, recentemente, Ludin et al. ${ }^{10}$ observaram uma correlação entre o fator de necrose tumoral, que está aumentado nos pacientes obesos, a fenda gengival e o índice de massa corporal.

Entretanto, Goodson et al. ${ }^{15}$ mostraram que a relação entre doença periodontal e obesidade poderia ser circunstancial, como o caso de dieta; oportuna, como a proliferação impulsionada por mudanças na atividade metabólica dos hospedeiros; e, também, causal, como na iniciação ou propagação da doença.

Além de correlacionar-se com a doença periodontal, a obesidade, de acordo com alguns estudos, está associada à cárie dentária ${ }^{8,22}$. Alguns estudos demonstram que a saúde bucal e a nutrição têm uma relação bidirecional ${ }^{22}$. A relação causal entre 0 consumo de açúcar e a cárie dentária pode ser comprovada por uma gama de estudos epidemiológicos e clínicos em humanos, por experimentos em animais, bem como por estudos do $\mathrm{pH}$ da placa bacteriana e estudos laboratoriais in vitro ${ }^{9}$. Dentre os açúcares da dieta, a sacarose tem sido apontada como o principal fator etiológico da cárie dentária, atuando como substrato para a produção de ácidos pelas bactérias cariogênicas, com subsequente desmineralização do esmalte dental, e também para a obesidade ${ }^{9}$.

Ao contrário dos autores citados, Pérez et al. ${ }^{12}$ associaram, por meio de um estudo longitudinal desenvolvido no período de quatro anos, cárie dental e IMC em 110 crianças de escola pública localizada no México. As crianças acima do peso apresentavam mais dentes erupcionados e menor índice de cárie em relação às demais.

Por sua vez, Karp ${ }^{31}$ relata a existência de uma relação entre cárie dentária e fatores de risco de doenças cardiovasculares em crianças obesas. Obesidade e cárie dentária tendem a aumentar conjuntamente, sobretudo devido ao fato de o aumento do consumo de açúcar estar relacionado a ambas as condições ${ }^{5}$.

Conforme demonstraram outros estudos, a cárie dentária está associada com o consumo de açúcar, que é o mesmo fator etiológico da obesidade, e não com a obesidade propriamente. Tramini et al..$^{22}$, objetivando analisar a associação entre IMC e CPOD, realizou um estudo com 835 alunos franceses com 12 anos de idade. O IMC médio foi de 18,9 para a amostra total e de 1,47 para valores correspondentes ao CPOD. Os resultados mostraram uma associação significativa entre CPOD e o consumo de açúcar, mas não com o IMC. Do mesmo modo, Moreira et al. ${ }^{8}$, por meio de um estudo transversal, propôs medir a associação entre cárie dentária e obesidade em 1.665 adolescentes obesos e em 1.665 com peso normal na faixa de 12 a 15 anos de idade que frequentavam escolas públicas e privadas. O índice de CPOD foi maior nas escolas públicas tanto para o grupo dos obesos quanto para os considerados de peso normal. Assim, concluiu-se em ambos os trabalhos que, dentro dos limites de um estudo transversal, não há associação significativa entre cárie dentária e obesidade.

Cinar e Murtomaa ${ }^{13}$, por meio de um estudo transversal, procuraram relações entre obesidade, saúde bucal e fatores de estilos de vida em 611 crianças entre 10 e 12 anos de escolas públicas e privadas em Istambul, Turquia. Tomaram como base questionários que visavam a obter dados da saúde bucal das crianças e de suas mães. As análises mostraram que é preciso tratar obesidade, saúde oral e nutrição em conjunto, favorecendo, assim, a promoção de saúde para melhorar o bem-estar dos mais jovens e capacitá-los a um bom estilo de vida.

Rossow et al. ${ }^{32}$ estudaram o padrão de consumo de açúcar na primeira infância em crianças norueguesas. Concluíram que o hábito de consumo é estabelecido já na infância, com padrões de aumento desde o nascimento até os dois anos de idade. 
Tal padrão mantém-se durante os cinco primeiros anos de vida ${ }^{5}$, justamente o que Freire et al. ${ }^{9}$ encontraram em seu estudo, objetivando investigar os hábitos dietéticos de um grupo de acadêmicos de Odontologia em relação ao açúcar, ao analisar a influência dos conhecimentos adquiridos durante o curso. Sessenta por cento dos formandos relataram ter reduzido a ingestão após o ingresso no curso, e a maioria pretende recomendar restrição de açúcar aos próprios filhos $(97,5 \%)$ e aos futuros pacientes (87\%), visando a uma melhor saúde bucal e geral $(77,0 \%)$. Concluiu-se que os conhecimentos adquiridos não levam, necessariamente, a uma mudança de hábitos dietéticos entre os acadêmicos de Odontologia, apesar de influenciarem em sua intenção de conduta junto aos filhos e pacientes, no futuro.

Uma revisão dos guias dietéticos internacionais lançados desde a década de 1960 demonstrou que a maioria $(84,5 \%)$ recomenda a redução do consumo de açúcares, tendo como objetivo a prevenção de várias doenças crônicas, principalmente a cárie e a obesidade ${ }^{9}$.

Os pacientes obesos apresentam qualidade de vida reduzida e necessitam de uma maior atenção quanto à saúde bucal, especialmente no que se refere à doença periodontal e à cárie dentária, para evitar que os problemas agravem-se. Os profissionais da saúde devem auxiliar e conscientizar a população em relação aos efeitos dos hábitos alimentares inapropriados sobre a saúde geral da população, assim como sobre a saúde bucal. Além disso, não devem centralizar o foco das atividades educativas somente nos riscos relacionados aos problemas bucais, mas estabelecer uma estratégia de ação que englobe fatores de risco comuns para a obesidade e as doenças da boca.

\section{Considerações finais}

Esta revisão de literatura demonstrou que ocorre uma interação entre a obesidade e a saúde bucal, principalmente quanto à cárie dentária e à doença periodontal. Entretanto, a maior parte dos estudos encontrados foi do tipo transversal, necessitando de mais estudos longitudinais para melhor demonstrar essa interação e, também, para poder eliminar alguns fatores de confusão que podem estar presentes nessa relação.

\section{Abstract}

Prevalence and incidence rates regarding obesity have become alarming in both adults and children. The excessive fat buildup in the body may lead to health problems of individuals. Objective: this study aims to present a literature review on the interaction between obesity and oral health from a study ordered in journals of national and international circulation. Materials and method: five hundred and fourteen (514) studies were found, among which 18 were selected based on the following criteria: time of publication, proper subject to the proposal of this work, clear, objective and consistent ideas for the title of the study. Results: the selected studies have shown associations between periodontal disease and obesity. There were differences regarding the work that linked obesity to dental caries, but those were the minority. Conclusion: it is concluded that obese patients require oral health care, especially concerning prevention of periodontal disease and dental caries.

Keywords: Oral health. Obesity. Periodontitis. Dental caries.

\section{Referências}

1. Kopelman PG. Obesity as a medical problem. Nature 2000; 404 (6778):635-43.

2. Merrouche M, Sabate JM, Jouet P, Harnois F, Scaringi S, Coffin B, et al. Gastro esophageal reflux and esophageal motility disorders in morbidly obese patients before and after bariatric surgery. Obes Surg 2007; 17(7):894-900.

3. World Health Organization (WHO). Oral health surveys: basic methods. Geneva: OMS; 1997.

4. Ritchie CS. Obesity and periodontal disease. Periodontol 2000 2007; 44:154-63.

5. Traebert J, Moreira EAM, Bosco VL, Almeida ICS. Transição alimentar: problema comum à obesidade e à cárie dentária. Rev Nutr 2004; 17(2):247-53.

6. Bezerra BB, Sallum EA, Sallum AW. Obesity and periodontal disease: why suggest such relationship? An overview. Bras Jl Oral Sci 2007; 6(23):1420-2.

7. Prpić J, Kuiš D, Pezelj-Ribarić S. Obesity and oral health - is there an association? Coll Antropol 2012; 36(3):755-9.

8. Moreira PVL, Rosenblatt A, Severo AMR. Prevalence of dental caries in obese and normal-weight Brazilian adolescents attending state and private schools. Community Dent Health; 2006; 23(4):251-3.

9. Freire MCM, Dias HRP, Sousa CS. Hábitos e atitudes dos acadêmicos de Odontologia da Universidade Federal de Goiás em relação ao açúcar e à saúde. Rev Odontol Univ São Paulo 1997; 11(3):221-7.

10. Ludin M, Yucel-Lindberg T, Dahllof G, Marcus C, Modeer T. Correlation between TNF- $\alpha$ in gingival fluid and body mass index in obese subjects. Acta Odontol Scand 2004; 62:273-7.

11. Castilhos ED, Horta BL, Giagante DP, Demarco FF, Peres KG, Peres MA. Association between obesity and periodontal disease in oung adults: a population-based birth cohort. J Clin Periodontol 2012; 39:717-24

12. Pérez LS, Irigoyen M, Zepeda M. Dental caries, tooth eruption timing and obesity. Acta Odontol Scand 2010; 68:57-64.

13. Cinar AB, Murtomaa H. Interrelation between obesity, oral health and life-style factors among Turkish school children [online]. Clin Oral Invest 2010. Disponível em URL: http:// www.springerlink.com/content/3n22124065r3752/fulltext. pdf. 
14. de Andrade FB, de Franca Caldas A, Jr., Kitoko PM. Relationship between oral health, nutrient intake and nutritional status in a sample of Brazilian elderly people. Gerodontol $2009 ; 26: 40-5$.

15. Goodson JM, Groppo D, Halem S, Carpino E. Is obesity an oral bacterial disease? J Dent Res 2009; 88:519-23.

16. Haffajee AD, Socransky SS. Relation of body mass index, periodontitis and Tannerella forsythia. J Clin Periodontol 2009; 36:89-99.

17. Jürgensen N, Petersen PE. Oral health and the impact of socio-behavioural factors in a cross sectional survey of 12 -year old school children in Laos. BMC Oral Health 2009; 9:29.

18. Khader YS, Bawadi HA, Haroun TF, Alomari M, Tayyem RF. The association between periodontal disease and obesity among adults in Jordan. J Clin Periodontol 2009; 36:18-24.

19. Kongstad J, Hvidtfeldt UA, Gronbaek M, Stoltze K, Holmstrup P. The relationship between body mass index and periodontitis in the copenhagen city heart study. J Periodontol 2009; 80(8):1246-53.

20. Kushiyama M, Shimazaki Y, Yamashita Y. Relationship between metabolic syndrome and periodontal disease in Japanese adults. J Periodontol 2009; 80(10):1610-5.

21. Morita T, Ogawa Y, Takada K, Nishinoue N, Sasaki Y, Motohashi M, et al. Association between periodontal disease and metabolic syndrome. J Public Health Dent 2009; 69(4):248-53.

22. Tramini P, Molinari N, Tentscher M, Demattei C, Schulte AG. Association between caries experience and body mass index in 12-year-old French children. Caries Res 2009; 43(6):468-73.

23. Ekuni D, Yamamoto T, Koyama R, Tsuneishi M, Naito K, Tobe KJ. Relationship between body mass index and periodontitis in young Japanese adults. Periodontal Res 2008; 43(4):417-21.

24. Hilgert JB, Hugo FN, de Sousa M da L, Bozzetti MC. Oral status and its association with obesity in Southern Brazilian older people. Gerodontology 2009; 26(1):46-52.

25. Ngatia EM, Gathece LW, Macigo FG, Mulli TK, Mutara LN, Wagaiyu EG. Nutritional and oral health status of an elderly population in Nairobi. East Afr Med J 2008; 85(8):378-85.

26. Östberg AL, Nyholm M, Gullberg B, Råstam L, Lindblad $\mathrm{U}$. Tooth loss and obesity in a defined Swedish population. Scand J Public Health 2009; 37(4):427-33.

27. Yöslato P, Suominen-Taipale L, Reunanen A, Knuuttila M. Association between body weight and periodontal infection. J Clin Periodontol 2008; 35(4):297-304.

28. Saito T, Shimazaki Y, Kiyohara Y, Kato I, Kubo M, Iida M, et al. Relationship between obesity, glucose tolerance, and periodontal disease in Japanese women: the Hisayama study. J Periodontal Res 2005; 40(4):346-53.

29. Al-Zahrani MS, Bissada NF and Borawskit EA. Obesity and periodontal disease in young, middle-aged, and older adults. J Periodontol 2003; 74(5):610-5.

30. Wood N, Johson RB, Streckfus CF. Comparison of body composition and periodontal disease using and periodontal disease using nutritional assessment techniques: Third National Health and Nutrition Examination Survey (NHANES III). J Clin Periodontol 2003; 30:321-7.

31. Karp WB. Childhood and adolescent obesity: a national epidemic. Calif Den Assoc J 1998; 26(10):771-3.
32. Rossow I, Kjaernes U, Holst D. Patterns of sugar consumption in early childhood. Com Dent Oral Epidemiol 1990; 18:12-6

\section{Endereço para correspondência:}

Silvia Helena de Carvalho Sales Peres Departamento de Odontopediatria, Ortodontia e Saúde Coletiva, Faculdade de Odontologia de Bauru da Universidade de São Paulo. Al. Octávio Pinheiro Brisolla, 9-75

17012-901 Bauru, SP

Fone: + 551432358256 . Fax + 55143235860

E-mail: shcperes@usp.br

Recebido: 10/08/2012. Aceito: 16/08/2013. 